\title{
Trek au Toit du Monde - Pamir chinois et Pakistan du Nord
}

Jean Martin

Colin, 6 ans, vit avec ses parents (papa suisse et maman américaine), son frère Dimitri, 4 ans, et sa toute petite sœur Ella, née en septembre 2007, dans le New Jersey, aux Etats-Unis. L'auteur réalise que Colin aura besoin d'une certaine assistance pour tout comprendre!

Correspondance:

Dr Jean Martin

La Ruelle 6

CH-1026 Echandens

jean.martin@urbanet.ch
Mon cher Colin*,

Je te fais ce message pour raconter ce que j'ai vu en Asie centrale durant trois semaines (11 juillet au 2 août 2007), au nord du Pakistan et dans la province occidentale de la Chine, le Sin-kiang; dans une région dénommée Pamir et qu'on appelle aussi le Toit du Monde. L'Asie centrale comporte les plus hautes montagnes de notre planète, en particulier 14 sommets de plus de 8000 mètres: la région de l'Himalaya et de chaînes de montagnes voisines qui s'appellent le Karakorum (où nous étions), les Kunlun, l'Hindu Kuch en limite de l'Afghanistan, ainsi que les Monts Célestes (Tian Shan) plus au nord.

\section{Les Routes de la Soie et leurs caravanes}

Cette région est fameuse parce qu'elle était traversée par la Route de la Soie: pendant près de deux mille ans, avant que les voyages par mer ne soient courants, on y voyait de grandes caravanes de chameaux; chameaux à deux bosses dits de Bactriane, différents des chameaux à une bosse, les dromadaires, qu'on voit dans les déserts d'Afrique et d'Arabie. Ces caravanes allaient de l'Est de la Chine (ville de Xi'an) jusqu'à la mer Méditerranée (qui elle est près de la Suisse, que nous verrons une fois ensemble) et vice versa. En fait, on devrait parler des Routes de la Soie parce qu'il y avait plusieurs trajets majeurs: pour aller d'est en ouest mais aussi du nord au sud. Un parcours très important reliait la Chine occidentale au sous-continent indien (Inde et Pakistan actuels, par le Cachemire); c'est une partie de cette route que nous avons suivie en fin de voyage.

Ces caravanes transportaient de la soie, tissu très léger, souple, soyeux, solide, diverses épices pour assaisonner la cuisine, des tapis, des parfums, des pierres précieuses, de la porcelaine de Chine, etc. A l'Ouest, on raffolait de certaines de ces denrées au temps de l'Egypte ancienne et de l'Empire romain déjà. Et les caravanes repartaient vers l'Orient (ou vers le nord) avec d'autres marchandises. Ce devait être extraordinaire de les voir avancer de leur pas chaloupé, posément, dans les zones cultivées, dans d'autres très arides, dans les montagnes.
Avant d'arriver à destination, il y avait des obstacles importants:

- D'abord, les Routes de la Soie traversaient des déserts, où il y a très peu d'eau et d'herbe pour les chameaux - et rien à manger pour les humains, où il fait terriblement chaud. On marche alors très tôt le matin ou même durant la nuit, et on se repose, en cherchant un coin d'ombre, durant la journée.

- Un autre obstacle était précisément les montagnes où le groupe dont je faisais partie s'est promené, avec des cols très hauts à 4000 et 5000 mètres d'altitude. Impassables en hiver.

- Là, il faisait froid (en montagne en général, on a bon chaud quand il faut beau et qu'il y a du soleil mais, dès que le temps se gâte, qu'il y a du vent, de la pluie ou de la neige - un peu comme quand nous sommes redescendus ensemble de l'Hôtel Weisshorn en juillet dernier - on a vite très froid et il faut être bien équipé). On raconte l'histoire d'une grande caravane de centaines de personnes et de chameaux, passant tard dans la saison, qui a été bloquée et anéantie par la neige et le froid, dans un plateau-cuvette (de Tchitchiklik) où nous avons campé.

- A l'époque, un obstacle encore était les brigands: des gens des régions traversées qui, à cheval, tombaient sur les caravanes et leur volaient leurs marchandises - et parfois tuaient les chameliers et commerçants ou les emmenaient comme esclaves. Une époque rude, comme tu vois.

\section{En Asie centrale}

La vie n'était donc pas du tout facile mais elle avait aussi des côtés riants: dans ces pays, on aimait beaucoup la poésie, faisait de la musique. On y confectionne de très bonnes pâtisseries. On y cultivait de la vigne et appréciait le vin; il y a encore de la vigne (grande production de raisins secs), mais pas vraiment de vin dans la mesure où la religion musulmane, celle de la région, ne permet pas de boire d'alcool. Il y avait des fêtes, religieuses ou pour les mariages, ou lors de grands rassemblements comme des foires foires aux animaux notamment. Un sport très 
particulier est ce qu'on appelle le rugby des nomades (bouzkachi): des cavaliers se disputent le corps d'une chèvre ou d'un mouton en se l'arrachant pour l'amener vers un but. Comme les sportifs dans nos pays, les meilleurs sont célèbres. J'ai aussi appris lors de ce voyage que le polo des officiers et aristocrates britanniques avait été adapté - et adopté - à partir d'un jeu qu'ils avaient découvert dans cette région, pratiqué dans la vallée de Hunza que j'évoque plus bas. ques sérieux - c'était à vrai dire une drôle d'impression de savoir que le risque existait que des combats armés s'intensifient à quelques kilomètres alors que tout était tranquille immédiatement autour de nous et que les gens étaient tout à fait amicaux. Nous avons visité des ruines et des musées, qui nous renseignent sur ce qui existait dans le passé, sur la manière dont les gens vivaient, dont ils mangeaient, élevaient des troupeaux, cultivaient la terre, construisaient des maisons et d'autres monuments, quels dieux ils

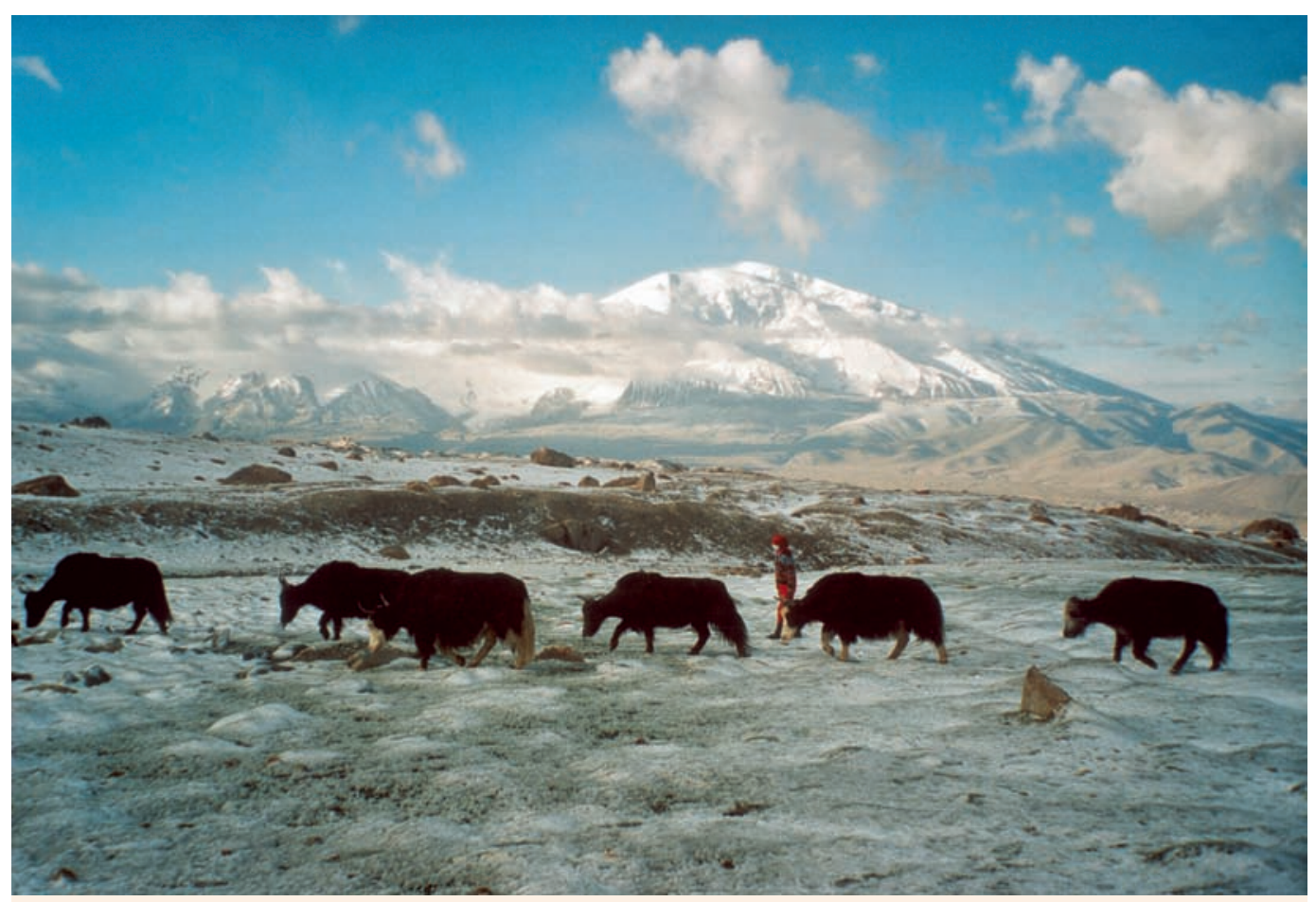

Au camp de base (vers 4500 m) du Mont Kongur, tôt le matin: une femme kirghize emmène ses yacks pâturer malgré la neige glacée tombée durant la nuit. Au fond, la masse du Mustagh Ata.

\section{Périple dans l'espace et le temps}

J'en viens à mon voyage: nous étions un groupe de 11 personnes, avec un guide qui savait beaucoup de choses sur les peuples de l'Asie centrale et leur histoire; y compris sur les grandes invasions de nomades qui, à plusieurs reprises au cours de l'histoire, sont venus jusqu'en Europe: des dizaines et centaines de milliers de cavaliers balayant tout sur leur passage ... Attila au $\mathrm{V}^{\mathrm{e}}$ siècle après Jésus-Christ, Gengis Khan au XIII ${ }^{\mathrm{e}}$ siècle. Tamerlan (Timur le boiteux, devenu le héros national de l'Ouzbékistan [1]) à la fin du XIVe notamment.

D'abord, nous avons volé de Paris jusqu'à Doha, au Qatar (dans la péninsule Arabique), puis de là à Islamabad, capitale du Pakistan. Ce pays était agité en juillet par des troubles politi- adoraient. Au IVe siècle avant Jésus-Christ, cette région a été conquise par Alexandre le Grand, venu de Grèce - plus exactement de Macédoine -, donc d'Europe, qui en quelques années a conquis un immense empire, jusqu'au fleuve Indus. Il a fondé sur son parcours une dizaine de villes qu'il a toutes appelées Alexandrie. Et on dit que, dans telle vallée reculée du Pakistan, vit une population qui descend des soldats d'Alexandre. Le Pakistan, aujourd'hui musulman, a aussi connu dans l'Antiquité une très belle civilisation bouddhique, de Gandhara. C'est toujours très intéressant l'histoire, j'espère que tu seras du même avis quand tu l'étudieras.

Puis nous avons volé d'Islamabad à Kachgar, une ville et un marché très important des Routes de la Soie, un «nœud», à l'extrême ouest de la 


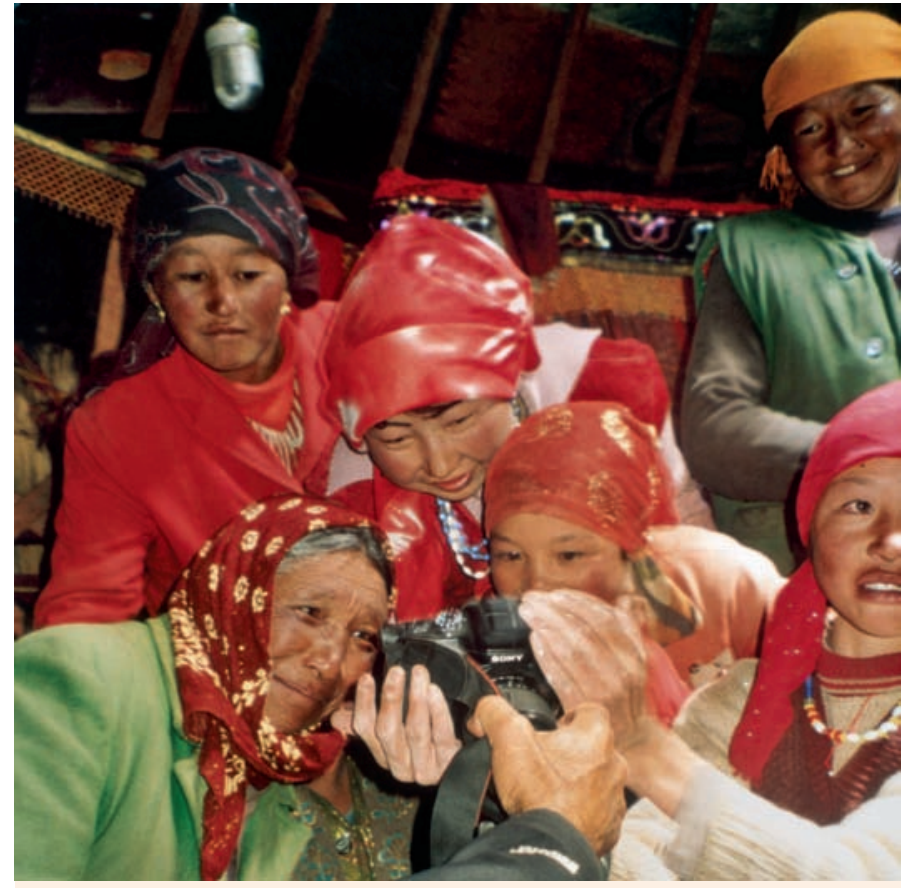

Dans une yourte kirghize de la vallée de Yango: les habitantes se passionnent pour leur image photographiée.

Chine - province du Sin-kiang. Quand on venait de Chine, c'est après Kachgar qu'on devait passer les hauts cols du Pamir ou du Karakorum, pour continuer vers l'ouest ou aller vers le sud. Et quand on venait d'Europe, on avait fait l'essentiel quand on arrivait à Kachgar; même s'il y avait encore un grand désert, le Takla-Makan, qu'il fallait contourner par le sud ou le nord avant d'arriver en Chine proprement dite. Nous y avons vu un grand marché aux animaux: chèvres, moutons, vaches, chevaux, ânes, qui était spectaculaire - Dimitri et toi auriez été très intéressés. Pas dans ce marché de chameaux et de yacks (le yack est une sorte de vache qui vit et se plait en haute montagne, à 4000 m et au-dessus), mais nous en verrons beaucoup durant notre marche. Note que le yack est le seul animal qui peut avancer avec des charges quand il y a beaucoup de neige (disons, plus de $30 \mathrm{ou} 40 \mathrm{~cm}$ ); les yacks remplaçaient donc parfois les chameaux dans les caravanes.

\section{Trek autour du Mustagh Ata}

Ensuite, nous avons entrepris une marche à pied de 11 jours, en restant en général entre 4000 et 4900 mètres d'altitude, qui nous a fait tourner autour d'un haut sommet du Pamir chinois, le Mustagh Ata - ce nom veut dire la MontagneMère (ou -Père) couverte de glace. C'est effectivement un immense dôme de glaciers, magnifique.
Il culmine à $7546 \mathrm{~m}$ et son voisin, le Kongur, que nous voyions aussi très bien, à $7719 \mathrm{~m}$. A la fin du trek, nous sommes passés par le camp de base du Kongur - un camp de base est l'endroit où les alpinistes se préparent pour partir à l'assaut d'une montagne; pour notre part, nous ne sommes pas allés plus haut!

Nous marchions dans de hautes vallées, passant de l'une à l'autre par des cols à près de $5000 \mathrm{~m}$. C'est un peu drôle parce que c'est l'altitude de la plus haute montagne d'Europe, le Mont-Blanc - que l'on voit bien depuis Morges. A ces hauteurs, certaines personnes ont de la peine à souffler ou peuvent souffrir de mal des montagnes. Un des nôtres, pourtant très bon coureur à pied sur de longues distances, a été vraiment mal fichu durant quatre ou cinq jours; ce qu'on observe en effet, c'est que le fait d'être entraîné physiquement ne protège pas du mal d'altitude - lié à une sensibilité individuelle.

Nous marchions environ cinq heures par jour, c'était assez astreignant. Avec nous, quinze chameaux (!) pour porter notre matériel et notre subsistance. En plus du guide français, nous avions un guide local sympathique, Wuarap, cinq chameliers et deux cuisiniers. L'avantage d'avoir des chameaux, c'est que si l'un de nous était trop fatigué, il pouvait monter sur un chameau qui le transportait - une ou deux personnes en ont profité. Dans l'après-midi, nous montions nos tentes (comme la tente sous laquelle nous avons dormi ensemble dans le jardin d'Echandens, l'an dernier); nous nous reposions, nous lisions, discutions entre nous et un peu avec les habitants du lieu - mais ce n'était pas facile, il fallait un interprète. En général, nous nous arrêtions dans un petit village, plutôt un hameau: quelques maisons de terre battue, parfois de grandes tentes de feutre qu'on appelle yourtes, avec quelques dizaines de personnes. Ni routes ni voitures, ici et là un poste de télévision. Pratiquement pas de choses modernes donc - à l'exception parfois surprise! - de panneaux solaires sur le toit des maisons, ce qui donnait un peu d'électricité et permettait d'avoir de la lumière le soir.

Bien que nous ayons été en plein été, il ne faisait pas trop chaud à ces hauteurs, c'était agréable. A deux reprises, nous avons trouvé de la neige dehors en nous réveillant le matin et il faisait froid. Neige qui fondait dans la journée.

\section{Des animaux et (pas partout) des fleurs}

Des quantités de marmottes. Celles de là-bas sont jaune-brun alors que dans les Alpes elles sont gris-brun. Elles crient de manière stridente quand elles nous voient arriver puis se cachent dans leurs terriers (comme vos groundhogs). Il y a 
dans ces régions un bouquetin (ibex siberica); nous n'en avons pas vu mais, par contre, avons trouvé sur le chemin, presque chaque jour, de belles cornes de ces bouquetins (peut-être les avaient-ils perdues en se battant ou parce qu'ils avaient été attaqués par un autre animal, je ne sais pas). Avons observé de grands oiseaux de proie, l'aigle et le gypaète barbu. Le gypaète est un vautour qui se nourrit d'animaux morts, il peut même manger leurs os: pour cela, il monte haut dans le ciel et laisse tomber les os sur des rochers où ils se cassent, ce qui permet à l'oiseau de manger la moelle à l'intérieur de l'os; ce gypaète a été réintroduit en Suisse et en France récemment et on en observe dans les Alpes. Quelques couples d'oies du Tibet, qui ressemblent aux oies du Canada qu'on voit chez vous. Aussi des cailles/perdrix des neiges et passablement de petits oiseaux, certains aux belles couleurs. Quelques lièvres. Quelques lézards. Puis bien sûr des troupeaux de yacks, de chèvres et de moutons. Et des fleurs: pas toujours mais tout d'un coup, à certains endroits, on trouvait un tapis coloré et diversifié de fleurs des montagnes. Ici et là, beaucoup d'edelweiss! Pas d'arbres à ces altitudes.

Ce qui me plaît dans une telle région, c'est d'admirer d'immenses montagnes couvertes de neige et de glaciers: vraiment impressionnantes (surtout quand on regarde avec les jumelles comme j'aime le faire). Ce que j'aime aussi, c'est être loin de tout, «au milieu de nulle part» comme vous dites en anglais. Cela m'a rappelé des treks dans l'Himalaya proche où nous nous sommes promenés avec Grandmammie il y a des années - même une fois avec ton papa quand il avait trois ans -; nous avions alors demandé à un monsieur népalais de le porter sur son dos dans une hotte pendant notre voyage de quelques jours dans la région de Pokhara.

\section{Karakorum Highway et vallée de Hunza}

Après le trek, nous avons fait un très long trajet de plus de mille kilomètres, en minibus, de la Chine où nous étions pour retourner à Islama- bad, par une route appelée la Karakorum Highway. Elle passe par la magnifique vallée de Hunza, très cultivée grâce à de remarquables travaux d'irrigation comme on les connaît en Valais ou en Savoie, avec en particulier des quantités d'abricotiers. Les gens disent de cette route qu'elle est la Huitième merveille du monde; elle est récente, a été réalisée entre 1966 et 1980 par un effort conjoint (des efforts titanesques) de la Chine et du Pakistan. Des centaines de kilomètres de route creusée dans des parois de rocher très pentues, où on a souvent des éboulements de cailloux et de terre qui peuvent bloquer le passage, où d'imposants camions extraordinairement décorés circulent et se croisent au centimètre. De temps en temps, un véhicule tombe dans le fleuve, à pic, cent ou deux cents mètres plus bas ... Heureusement, nous n'en avons pas vu durant les trois jours où nous avons suivi cette route mais on comprend bien que cela puisse arriver.

Et puis, fatigués mais contents, avons repris l'avion à Islamabad tôt le matin du 2 août et sommes rentrés sur Paris, en survolant les déserts du Pakistan du Sud, de l'Iran et de l'Arabie (avec à nouveau une escale à Doha), puis en passant au dessus de l'île de Chypre, d'Istanbul et de Venise. J'ai passé la nuit dans un hôtel près de l'aéroport de Paris/Roissy et suis revenu à Genève et Echandens le lendemain.

Voilà, chef, je t'ai fait une bien longue histoire, peut-être est-elle tellement longue qu'elle te fatiguera. Tu peux essayer de la résumer à ton petit frère mais je crois qu'il est plus intéressé par ses jeux d'ordinateur - il a le droit. Et bien sûr, si tu veux me poser des questions sur ce que j'ai vu ou ce que je te dis, je tâcherai d’y répondre.

\section{Grandpa}

\section{Référence}

1 Martin J, Ouzbékistan en forme d'abécédaire De l'Antiquité à la Route de la Soie et à Tamerlan, au Grand Jeu et à l'assèchement de la mer d'Aral. Bull Méd Suisses. 2006;87(50):2193-8. 